INRA, Prod. Anim., 2005, 18 (2), 101-110

\title{
Les variations saisonnières des performances de reproduction chez la truie
}

\author{
H. QUESNEL 1 , S. BOULOT ${ }^{2}$, Y. LE COZLER 3 \\ ${ }^{1}$ INRA, UMR, Système d'Elevage, Nutrition Animale et Humaine, F-35590 Saint-Gilles \\ 2 ITP, Pôle technique d'élevage, F-35651 Le Rheu \\ 3 EDE, Chambre d'agriculture de Bretagne, Maison de l'Agriculture, F-56002 Vannes \\ Courriel : Helene.Quesnel@rennes.inra.fr
}

\begin{abstract}
Une baisse des performances de reproduction des truies en été et au début de l'automne affecte de nombreux élevages, en France comme à l'étranger. Elle se traduit par une diminution de la productivité numérique des truies, qui se répercute sur la production annuelle de porcs charcutiers. Au manque à gagner ainsi induit s'ajoute une plus grande variabilité des résultats techniques, difficile à gérer en élevage. L'infertilité saisonnière aggrave également le risque de réforme des truies. Quelles sont les causes de cette dépression «estivale» des performances de reproduction?
\end{abstract}

Contrairement à la laie ou à certains mammifères domestiques, la truie ne connaît pas de véritable anœstrus saisonnier. Cependant, une baisse des performances de reproduction des truies est observée en été et au début de l'automne dans de très nombreux pays, de l'Australie à la Finlande en passant par la France, les Etats-Unis ou la Thaïlande. Elle est connue sous le nom d'infertilité d'été ou, plus correctement, d'infertilité saisonnière. L’influence de la saison est attribuée aux deux caractéristiques premières des saisons, à savoir la lumière (photopériode et intensité lumineuse) et les températures ambiantes. Pourtant, les pays où sont rencontrées ces baisses saisonnières des performances présentent des climats variés, avec des variations annuelles thermiques et photopériodiques très différentes. La part relative de ces deux facteurs d'influence varierait donc d'un pays à l'autre.

Par ailleurs, des élevages d'une même zone géographique peuvent être différemment affectés par l'effet de la saison, certains étant peu touchés et d'autres subissant une baisse de performances très nette. La conduite d'élevage modulerait donc l'impact de l'été sur les performances de reproduction des truies.
Après avoir décrit l'impact de la saison sur les performances de reproduction des truies, nous étudierons les rôles respectifs de la photopériode et de la température ambiante ainsi que les mécanismes physiologiques impliqués. Pour aider à la compréhension de ces mécanismes, un bref rappel du contrôle endocrinien de la reproduction chez la truie sera présenté. Nous évoquerons également les autres facteurs qui modulent les manifestations d'infertilité saisonnière et en particulier le rôle du verrat.

\section{1 / Effets de la saison sur les performances de reproduc- tion des truies}

\section{1 / L'âge à la puberté}

La puberté des truies est retardée en été. Ainsi, le pourcentage de cochettes pubères à 7 ou 8 mois est plus faible en été que le reste de l'année (Christenson 1981, Paterson et Pearce 1990, Cronin et al 1983). Cependant, cet effet de l'été est partiellement voire totalement supprimé quand les cochettes sont élevées en présence d'un verrat sexuellement mature (Paterson et al 1991).

\section{2 / Le taux de mise bas}

La proportion de truies inséminées qui mettent bas (ou taux de mise bas) varie selon le mois d'insémination. Les services échographiques français rapportent une baisse systématique du taux de truies confirmées gestantes à 30 jours, entre juin et septembre. La dégradation des taux de gestation est de $5 \%$ en moyenne, mais elle a atteint $10 \%$ lors de la canicule de 2003 (Gènes Diffusion, communication personnelle). Une baisse du taux de mise bas est rapportée pour les inséminations de juin à novembre dans le Minnesota et l'Iowa ( $-5 \%$, Xue et al 1994), de juillet à septembre en Angleterre (-5\%, Dawson et al 1998, dans des élevages de plein air), de juillet à octobre en Suède $(-8 \%$, Tummaruk et al 2001), d'août à octobre en Finlande (-7\%, Peltoniemi et al 1999) ou encore de décembre à février en Australie ( $-15 \%$, Hennessy et Williamson 1984). Cette dernière étude montre une évolution inverse des taux de mise bas et des températures ambiantes, et ce malgré une nette amélioration du taux de mise bas entre les années 75 à 77 et les années 79 à 81 (figure 1).

En élevage, le diagnostic de gestation est obtenu par détection d'éventuels retours en chaleur après insémination 
Figure 1. Evolution du taux de mise bas et de la température ambiante en fonction du mois d'insémination (Hennessy et Williamson 1984, Australie).

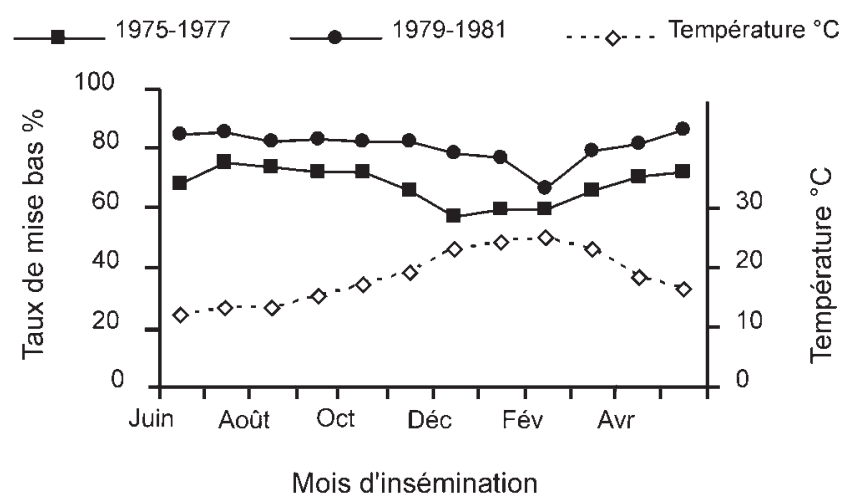

et/ou par échographie à ultrasons, généralement réalisée quatre semaines après l’insémination. Environ 50 \% des élevages français ont recours à l'échographie. Un retour en chaleur environ 21 jours après la saillie est dit «régulier» car il correspond à la durée d'un cycle sexuel normal. Il traduit un problème lié à l'insémination ou à l'établissement de la gestation (pas de fécondation ou forte mortalité embryonnaire avant implantation). Un retour en chaleur dans les 25 à 30 jours après insémination, dit «irrégulier», traduit l'interruption de la gestation après implantation des embryons par perte de la totalité de la portée. Aux Pays-Bas, Elbers et al (1994) observent que la fréquence de ces deux types de retours augmente significativement en été. Cependant, la majorité des études

montrent surtout une augmentation des retours irréguliers (Love et al 1993, Xue et al 1994, Koketsu et al 1997, figure 2), ce qui indique qu'une perte embryonnaire en deuxième quinzaine de gestation est la cause majeure de la baisse estivale du taux de mise bas. Les avortements plus tardifs, détectables par l'éleveur du fait de l'expulsion de fœtus de plus de 40 jours, augmenteraient également en début d'automne (Leman 1992).

\section{3 / La taille de la portée}

L’impact de la saison d'insémination sur la taille de la portée à la naissance est variable selon les études : peu ou pas de variations (Peltoniemi et al 1999, Tummaruk et al 2001) ou une diminution de 0,4 à 1 porcelet né vivant quand

Figure 2. Pourcentage de truies qui reviennent en oestrus après insémination en fonction du mois d'insémination (Xue et al 1994, USA). Retour en oestrus régulier : 18 à 25 jours après l'insémination ; Retour irrégulier : 26 à 37 jours après l'insémination.

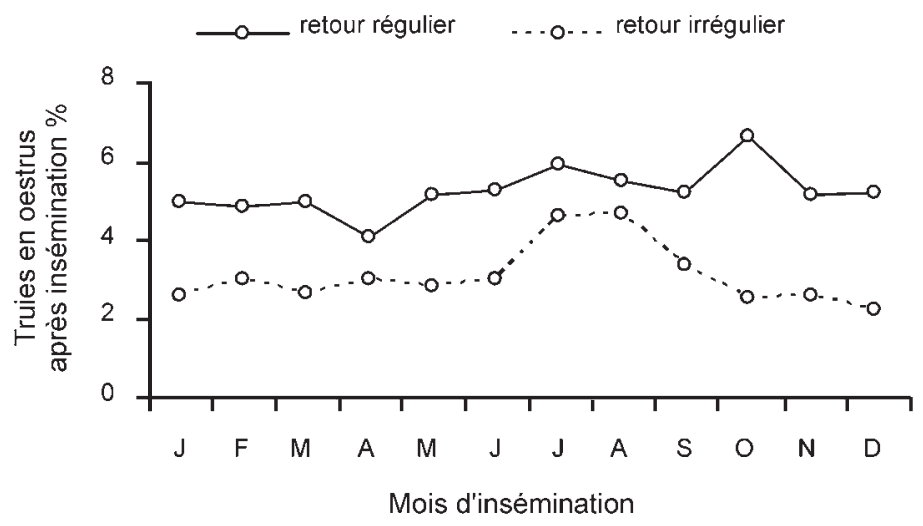

les truies sont inséminées en été ou début d'automne (Xue et al 1994, Dominguez et al 1996, Dawson et al 1998). Dans l'étude de Xue et al (1994), l'effet saison n'est apparent que chez les truies multipares, ce qui suggère que la taille de la portée est influencée par des événements survenant pendant la lactation précédant l’insémination.

Peu d'études décrivent les variations saisonnières du taux d'ovulation et les résultats sont contradictoires. Sur 415 truies abattues, Martinat-Botté et al (1984) observent un taux d'ovulation plus élevé en été et automne qu'en hiver et au printemps. Dans cette étude, la taille des portées évolue parallèlement au taux d'ovulation mais les variations saisonnières du nombre de porcelets nés vivants ne sont pas présentées. Pour Hochereau de Reviers et al (1997), les taux d'ovulation des cochettes présentent des variations saisonnières significatives, avec des valeurs maximales au printemps et minimales en automne.

\section{4 / L’intervalle sevrage-oestrus}

La durée moyenne de l'intervalle sevrage-oestrus des truies est allongée en été (tableau 1). Ces variations moyennes peuvent paraître faibles, notamment dans les études les plus récentes. En élevage, néanmoins, elles se traduisent par une augmentation de la proportion de truies présentant un intervalle sevrage-oestrus prolongé (Martinat-Botté et al 1984, Xue et al 1994). Un accroissement de la proportion de truies en anoestrus est également signalé en période estivale et en automne. Les effets de la saison sur l'expression de l'oestrus (durée, intensité) ou le moment de l'ovulation ont été peu étudiés (Steverink et al 1999). Selon Belstra et al (2004), pour une même durée de lactation, la durée de l'oestrus serait allongée de 8 heures et le moment d'ovulation retardé en été par rapport au printemps. Ceci serait théoriquement de nature à dégrader la fertilité, en affectant la qualité de détection des chaleurs et la détermina-

Tableau 1. Influence de la saison sur la durée moyenne de l'intervalle sevrage-oestrus (jours).

\begin{tabular}{|l|c|c|c|c|c|c|l|}
\hline \multicolumn{1}{|c|}{ Truies } & Effectif & Hiver & Printemps & Eté & Automne & Pays & \multicolumn{1}{|c|}{ Référence } \\
\hline Primipares & 36 & 3,9 & - & 9,3 & - & USA & Armstrong et al 1986 \\
& 170 & 3,8 & 11,1 & 10,7 & 7,7 & USA & Clark et al 1986 \\
& $>10000$ & 6,4 & 6,4 & 6,5 & 6,5 & U.K. & Dawson et al 1998 \\
\hline Multipares & 3119 & - & 7,0 & 10,6 & 6,7 & USA & Britt et al 1983 \\
& 236 & 5,7 & 4,9 & 5,3 & 6,8 & USA & Clark et al 1986 \\
\hline Confondus & 2948 & 7,7 & 8,2 & 8,1 & 7,5 & Pays-Bas & Vesseur et al 1994a \\
\hline
\end{tabular}


tion du moment optimal d’insémination. Toutefois les auteurs n'observent pas ces effets significatifs dans toutes les exploitations étudiées. Les truies qui ne sont pas inséminées en même temps que le reste de la bande reçoivent souvent un traitement hormonal.

La variation saisonnière de l'intervalle sevrage-oestrus est généralement plus marquée pour les truies primipares que pour les truies multipares (Karlberg 1980, Martinat-Botté et al 1984). Ceci n'est guère surprenant, car, d'une façon générale, les jeunes truies connaissent plus de problème d'anœstrus que les truies plus âgées, quelle que soit la saison. En effet, elles présentent plus de risques d'être en déficit nutritionnel pendant la lactation et elles ont moins de réserves corporelles pouvant tamponner l'impact du déficit (Dourmad et al 1994, Quesnel et Prunier 1995).

Des variations apparemment faibles de la durée de l'intervalle sevrageoestrus influencent les performances de reproduction des truies après le sevrage (Vesseur et al 1994b, Le Cozler et al 1997). Ainsi, le taux de gestation et la taille de la portée tendent à diminuer quand l'intervalle sevrage-oestrus varie de 4 à 8 jours. Les variations saisonnières de la fertilité et de la prolificité pourraient donc être en partie liées à l'impact de la saison sur le retour en oestrus. Cependant, les variations de durée de l'intervalle sevrage-oestrus ne suffisent pas à expliquer l'amplitude de la baisse estivale des performances.

\section{5 / La qualité de la semence}

Une partie des échecs de gestation observés en été pourrait être attribuée à des défauts de qualité de semence. En effet, le verrat est tout aussi sensible que la truie aux variations de la photopériode et aux effets négatifs des températures élevées (Levis 2002). En conséquence, la quantité et la qualité de la semence produite par les verrats présentent d'importantes variations saisonnières. Ainsi la viabilité et la motilité des spermatozoïdes sont plus faibles en été qu'en hiver (Guillouet et al 1999, Huang et al 2000, Ciereszko et al 2000, Park et Yi 2002). Néanmoins, les doses délivrées par les centres d'insémination artificielle satisfont un cahier des charges garantissant des caractéristiques constantes de la semence tout au long de l'année. Par contre, le risque de dégradation saisonnière de la qualité de la semence n'est pas nul dans les élevages qui produisent leurs doses ou qui pratiquent la saillie naturelle. Quelle que soit leur provenance, les doses de semence doivent être transportées et conservées à l'abri de la chaleur, les températures supérieures à $35^{\circ} \mathrm{C}$ pouvant définitivement altérer leur pouvoir fertilisant.

\section{6 / Conséquences globales}

La saison affecte donc les différentes phases de la reproduction chez la truie, avec des conséquences majeures sur le taux de mise bas. Les échecs de gestation et les anœstrus prolongés après le sevrage accroissent le risque de réforme. L'augmentation du taux de réforme observée en été et au début de l'automne touche plus sévèrement les jeunes truies, nullipares et primipares (Xue et al 1994). Les éleveurs pratiquent généralement la conduite en bandes et cherchent donc à synchroniser les cycles de reproduction des truies. Les échecs de gestation perturbent cette pratique et augmentent le nombre de jours improductifs. L'éleveur aura à décider s'il garde ces truies ou s’il les réforme, en fonction de nombreux critères (âge de la truie, nombre d'échecs, places disponibles...). Au coût direct des places vides en maternité s’ajoute donc celui d'un renouvellement plus important (réformes anticipées et accroissement des achats de cochettes). Les conséquences de ces moindres performances en été et au début de l'automne ne touchent pas que les truies. Avoir moins de porcelets par bande entraîne un surcoût de fonctionnement et un manque à gagner aux stades ultérieurs de l’élevage.

\section{2 / Rappel du contrôle en- docrinien de la reproduction}

\section{1 / Description du contrôle endocrinien de la reproduction}

L'activité des ovaires est contrôlée d'une part par les hormones gonadotro- pes, LH (hormone lutéinisante) et FSH (hormone folliculo-stimulante), et d'autre part par des boucles de rétrocontrôle faisant intervenir l'hypothalamus, l'hypophyse, les ovaires et l'utérus (figure 3). Les hormones gonadotropes sont sécrétées par l'hypophyse sous l'action d'un neuropeptide hypothalamique, le GnRH (Gonadotropin Releasing Factor). La sécrétion du GnRH, sous forme de pulses, est influencée par de nombreux facteurs détectés dans le système nerveux central qui intègre les informations issues de l'animal (état nutritionnel, métabolique, stress...) et de son environnement (lumière, température ambiante, disponibilité de nourriture...). Elle est également étroitement contrôlée par les stéroïdes sécrétés par les ovaires (Martinat-Botté et al 1996, Quesnel et al 1996).

L’ovulation nécessite une sécrétion élevée des hormones gonadotropes. Celles-ci stimulent la maturation et la croissance terminale des follicules (phase folliculaire du cycle sexuel). Ces follicules sécrètent des oestrogènes en grande quantité qui induisent d'une part le comportement d'oestrus et d'autre part, par un mécanisme de rétrocontrôle positif, la libération massive de LH qui déclenche l'ovulation. Les follicules qui viennent d'ovuler se transforment en corps jaunes qui sécrètent de la progestérone (phase lutéale). Cette hormone prépare l'utérus à une éventuelle gestation en limitant ses contractions. Environ 12 jours après l'ovulation, l'utérus sécrète des prostaglandines qui, en l'absence de fécondation, induisent la régression des corps jaunes et ainsi stoppent la sécrétion de progestérone. S’il y a eu fécondation, les embryons signalent leur présence dans l'utérus par un double signal oestrogénique : le premier signal, vers

Figure 3. Représentation schématique du contrôle endocrinien de la reproduction.

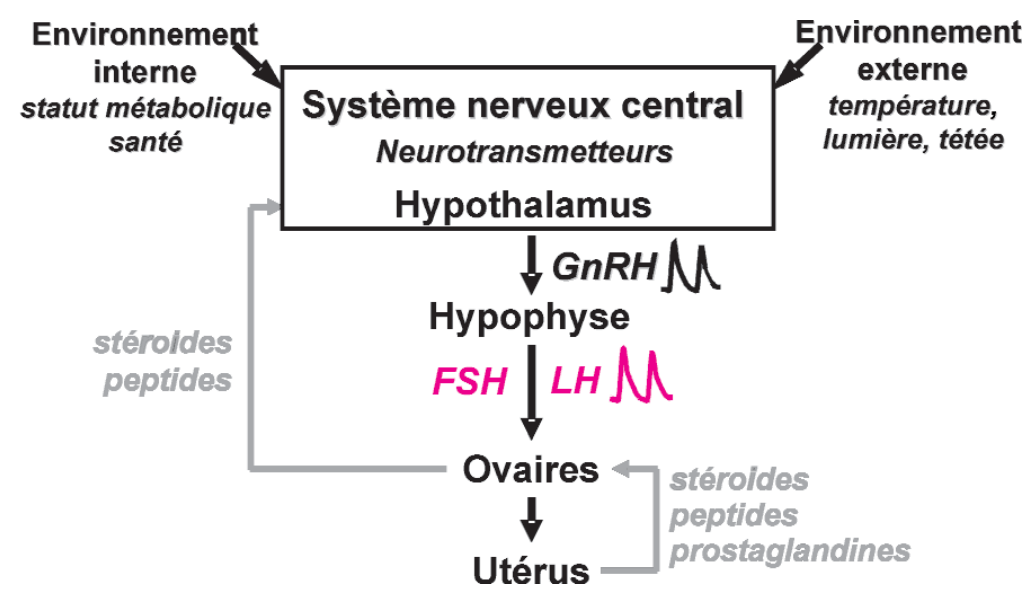


12 jours après fécondation, réoriente les prostaglandines vers la lumière utérine, et empêche ainsi la régression des corps jaunes. Le second signal, vers 18 jours, maintient la réorientation des prostaglandines vers l'extérieur. L'implantation des embryons dans la paroi utérine se fait entre 12 et 18 jours de gestation. La sécrétion de progestérone par les corps jaunes est stimulée par la LH maternelle pendant le premier mois de gestation, puis par la prolactine. Les concentrations de progestérone restent donc élevées tout au long de la gestation, ce qui maintient l'utérus dans un état quiescent.

Pendant la lactation, les réflexes neuroendocriniens induits par la tétée stimulent la sécrétion des hormones impliquées dans la lactogénèse et l'éjection du lait, mais inhibent la sécrétion du GnRH. Les truies sont donc généralement en anœstrus pendant toute la lactation. Si l'état catabolique que connaissent de nombreuses truies allaitantes, primipares notamment, est particulièrement sévère, il peut amplifier l'inhibition de l'axe de reproduction. Le sevrage des porcelets vers 21 ou 28 jours d’âge lève le frein imposé par la tétée, libérant ainsi les sécrétions de LH et de FSH et induisant la maturation finale des follicules. Les truies reviennent généralement en oestrus dans les 4 à 6 jours qui suivent le sevrage. Néanmoins, la reprise de l'activité ovarienne après le sevrage, qui conditionne la durée de l'intervalle sevrage-œstrus, dépend partiellement de l'ampleur de l'inhibition du GnRH pendant la lactation. Tous les facteurs qui affectent la physiologie et/ou l'état métabolique des truies pendant la lactation et aux alentours du sevrage peuvent donc influencer le retour en chaleur (Quesnel et Prunier 1995).

\section{2 / Impact de la saison}

L'activité de l'unité hypothalomohypophysaire présente des variations saisonnières. Chez des truies cycliques, les concentrations plasmatiques moyennes de LH sont maximales vers février et minimales vers août (Bassett et al 2001). Chez des truies primipares, les teneurs hypothalamiques de GnRH et les concentrations hypophysaires et sériques de LH aux alentours du sevrage sont plus faibles en été qu'en hiver, ce qui entraîne davantage d'anoestrus (Armstrong et al 1986). Cette altération de l'activité de l'unité hypothalomohypophysaire pourrait être due à une modification de sa sensibilité aux oestrogènes ovariens. En effet, en injectant de l'oestradiol-benzoate à des truies primipares ovariectomisées, Cox et al (1987) ont démontré que les truies répondaient moins au rétrocontrôle positif des oestrogènes en automne que pendant les trois autres saisons de l’année. Cette altération jouerait un rôle dans l'effet de la saison sur l'apparition de la puberté et de l'oestrus après le sevrage.

Pendant la gestation, les concentrations plasmatiques de progestérone sont plus faibles en automne qu'en hiver (Wrathall et al 1986), ce qui peut être dû à un signal lutéotrophe plus faible. En effet, la sécrétion de LH (concentrations moyennes, amplitude ou fréquence des pulses) pendant la gestation est également plus faible en été et au début de l'automne qu'en hiver (Peltoniemi et al 2000). Cette baisse de LH pourrait être amplifiée par la restriction alimentaire qui est souvent imposée en début de gestation par la conduite d'élevage. Les effets conjoints de la saison et de la nutrition sur la LH semblent insuffisants pour induire la régression des corps jaunes (Peltoniemi et al 2000). Par contre, la baisse des concentrations circulantes de progestérone qui en résulte limiterait l'aptitude des embryons à produire le deuxième signal oestrogénique vers 18 jours de gestation. La gestation est alors interrompue et suivie d'un retour «irrégulier» en oestrus, 25 à 30 jours après l’insémination.

D’après Tast et al (2002), cette baisse saisonnière des concentrations de progestérone n'est pas observée sur l'ensemble des truies mais seulement chez les femelles qui avortent. Il existerait donc des différences de sensibilité aux effets saisonniers, non encore élucidées. Le stress potentiellement induit par l'ensemble des facteurs associés à la période estivale (jours longs ou décroissants, températures élevées, déficits hydrique et nutritionnel...) a été évoqué comme un déterminant de la dépression saisonnière de fertilité (Hoste et Oakenfull 1999). Or la réponse de l'axe corticotrope au stress varie d'un animal à l'autre. En Australie, Wan et al (1994) ont montré que les truies et surtout les cochettes qui présentent une forte réactivité au stress, évaluée par la sécrétion de cortisol après injection d'ACTH, sont plus susceptibles de connaître des problèmes de reproduction en été.

\section{3 / Mécanismes impliqués dans l'effet saison}

Dans les zones tempérées, les saisons sont caractérisées essentiellement par la photopériode (évolution de la durée quotidienne d'éclairement) et les températures ambiantes. Dans les zones tropicales, deux saisons sont distinguées, essentiellement sur la base des températures et éventuellement de l'humidité.

\section{1 / La photopériode et l'inten- sité lumineuse}

Les effets propres de la durée d'éclairement et des températures élevées sont difficiles à dissocier sous nos latitudes. C'est moins le cas en Norvège, par exemple, où les températures maximales restent le plus souvent inférieures à $20^{\circ} \mathrm{C}$, alors que la photopériode présente des variations importantes à proximité du cercle polaire. Les performances de reproduction (âge à la puberté, retour en oestrus après le sevrage et taux de mise bas) sont plus faibles dans la région située au nord du cercle polaire, où l'évolution annuelle de la photopériode est extrême (24 heures de jour en juin et juillet), que dans la région située au sud du cercle polaire (Gaustad-Aas et al 2004).

L'existence d'une relation entre photopériode et fertilité est étayée dans plusieurs pays. Ainsi, les plus mauvais taux de mise bas sont observés pour les mises à la reproduction en août et septembre en Finlande (Peltoniemi et al 1999) et fin septembre en Amérique du Nord (Leman 1992), c'est-à-dire dans la période de l'année où la durée journalière d'éclairement diminue beaucoup et où les températures ambiantes ne sont pas les plus chaudes. A cette même période, les retours en chaleurs 25 à 35 jours après insémination deviennent plus nombreux que le reste de l'année.

L’influence de la photopériode sur l'oestrus et l'ovulation est démontrée expérimentalement. L'effet de l'été sur la venue en puberté est mimé en soumettant les cochettes à des durées d'éclairement longues (Flowers et al 1989). Dans une étude en photopériode contrôlée, où les durées d'éclairement sont progressivement prolongées (de 12 à $16 \mathrm{~h} / \mathrm{j}$ ) ou réduites (de 12 à $8 \mathrm{~h} / \mathrm{j}$ ) pendant la gestation et stabilisées pendant la lactation, la proportion de truies en oestrus dans les 10 jours post-sevrage est plus faible lorsque la durée d'éclairement est longue (figure 4, Prunier et al 1994). L’expérience a été réalisée en hiver et en été, avec des températures ambiantes dans les maternités variant de 20 à $25{ }^{\circ} \mathrm{C}$ en hiver et 25 à $35^{\circ} \mathrm{C}$ en été. 
Figure 4. Influence du mois de mise bas (janvier ou juillet) et de la durée d'éclairement (longue ou courte) sur le pourcentage de truies venues en oestrus après le sevrage (67 truies, Prunier et al 1994).

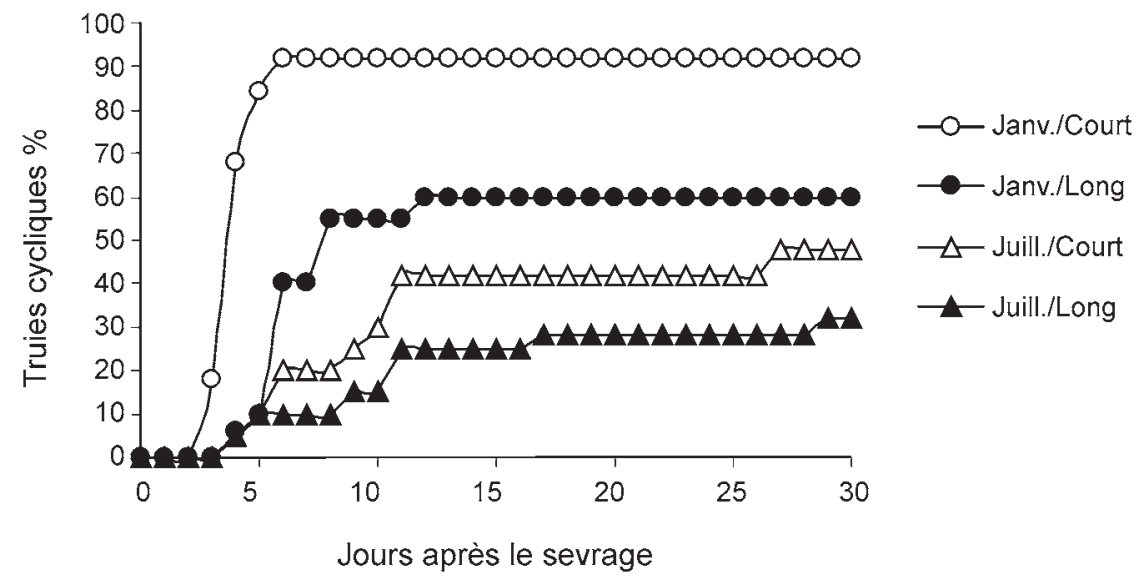

L’effet de la photopériode sur la durée moyenne des intervalles sevrageoestrus n'est significatif qu'en hiver, ce qui suggère une influence prédominante des températures ambiantes en été.

Les mécanismes qui sous-tendent l'effet de la photopériode sur l'activité sexuelle sont connus depuis longtemps dans les espèces à reproduction saisonnière. Le cerveau convertit les changements de durée d'éclairement en signal hormonal, via la sécrétion de mélatonine par la glande pinéale (Malpaux et al 2001). Cette hormone est secrétée pendant la phase obscure. Quand les jours sont longs, la sécrétion de mélatonine est de courte durée et l'activité de reproduction est interrompue chez les espèces dites «de jours courts». Une sécrétion de mélatonine de longue durée est interprétée comme un jour court et active l'activité sexuelle chez ces espèces. Ainsi, chez la laie et le sanglier, l'activité sexuelle est interrompue de juin à septembre, pendant les jours longs (12 heures et plus d'éclairement quotidien), et commence à reprendre quand la durée de la photophase devient inférieure à la durée de la phase obscure (à partir de l'équinoxe d'automne). L'existence d'un pic de sécrétion de mélatonine pendant la nuit a longtemps été controversée chez la truie. Cependant, des études récentes ont démontré que la mélatonine était sécrétée selon un rythme journalier chez le porc domestique comme chez le sanglier, avec une augmentation des concentrations plasmatiques pendant la phase obscure (Tast et al 2001). Il en résulte des variations annuelles des teneurs plasmatiques en mélatonine (tableau 2, Tast et al 2001). Les implants de mélatonine peuvent lever l'anœstrus saisonnier chez la brebis

a, b $P<0,01$.
(Chemineau et al 1996). Dans une étude comportant une vingtaine de truies sélectionnées pour avoir manifesté des baisses de performances en été lors des années précédentes, et élevées en plein air en l'absence de verrat, Bassett et al (2001) ont montré que la pose d'implants de mélatonine à l'équinoxe du printemps, dans le but de mimer les conséquences des jours courts, permet d'éviter l'anoestrus saisonnier chez ces truies. Cependant, l'évolution dans le temps des concentrations plasmatiques de LH ne diffère pas entre truies traitées et truies témoins. Le ou les liens physiologiques entre mélatonine et reproduction restent donc à éclaircir.

Des programmes lumineux permettant d'éviter les jours longs ont été testés en élevage, notamment en Allemagne (Claus et al 1984, Levis 2002). Dans deux élevages, l'intervalle sevrage-oestrus était nettement détérioré en été (supérieur à 20 ou 30 jours). L'année suivante, le programme lumineux a imposé une baisse progressive de la durée d'éclairement pendant la gestation et la lactation (-20 minutes par semaine), de mai à août. L'intervalle sevrage-oestrus moyen en été a été inférieur à 6 jours. Dans ces essais, l'absence de véritables animaux témoins, contemporains des animaux sous programme lumineux, limite toute conclusion sur l'efficacité de tels programmes.

Le fait que la période de dépression de fertilité coïncide avec une forte diminution de la durée quotidienne d'éclairement (fin d'été, début d'automne) conduit souvent à penser sur le terrain que les photopériodes décroissantes, et par extrapolation les photopériodes courtes, ont un effet négatif sur la reproduction. Certains auteurs recommandent de ce fait des photopériodes longues de 12 à 16 heures en gestation par exemple (Muirhead et Alexander 2000). Cette confusion conduit aussi à recommander d'éclairer longtemps, voire 24 heures sur 24, les truies après le sevrage pour stimuler la venue en chaleur. A notre connaissance, rien n'atteste de l'efficacité d'une telle pratique.

\section{2 / La température ambiante}

Lorsque la température ambiante augmente au-delà de la zone de confort thermique, les animaux homéothermes mettent en place des adaptations pour lutter contre l'élévation de leur température corporelle. Le mécanisme le plus adapté chez le porc est la baisse de la consommation d'aliment qui permet de réduire la production de chaleur interne liée à l'utilisation digestive et métabolique des aliments (ou «extra-chaleur»). Quand la température ambiante dépasse la limite supérieure de la zone de thermoneutralité (ou température critique supérieure), les adaptations mises en place ne suffisent plus et la température interne augmente. Les températures critiques supérieures sont comprises entre 25 et $32^{\circ} \mathrm{C}$ pour la truie en gestation et entre 22 et $25^{\circ} \mathrm{C}$ pour la truie en lactation.

En élevage, la mortalité des truies augmente en été lorsque la température ambiante monte (Xue et al 1994, D’Allaire et al 1996). Ces mortalités sont principalement dues à des problèmes cardiaques, plus fréquents au-delà de $30^{\circ} \mathrm{C}$.

Différentes techniques permettent de rafraîchir les truies (air frais, aspersion par goutte à goutte) ou l'ambiance des maternités («cooling» : l’air extérieur passe par une maille imbibée d'eau). Les effets du cooling sur la consomma-

Tableau 2. Sécrétion nocturne de mélatonine chez le sanglier et la cochette domestique : durée moyenne sur 24 heures (en heures) aux équinoxes de printemps et d'automne et aux solstices d'été et d'hiver en Finlande (Tast et al 2001a).

\begin{tabular}{|l|c|c|c|c|c|}
\hline & Effectif & Printemps & Eté & Automne & Hiver \\
\hline Sanglier & $4-6$ & 10 & $6 \mathrm{a}$ & 11 & $17 \mathrm{~b}$ \\
\hline Cochette & $4-6$ & 9 & $8 \mathrm{a}$ & 12 & $11 \mathrm{~b}$ \\
\hline
\end{tabular}


tion et les performances des truies sont variables (Tubbs 1990, Peltoniemi et al 2000). Les techniques dirigées vers les femelles améliorent de 20 à 90 \% la consommation de truies exposées à $30^{\circ} \mathrm{C}$ (McGlone et al 1988). Cependant, comme ces techniques augmentent le taux d'humidité de l'air, elles peuvent avoir un effet inverse à l'effet recherché (Rozeboom et al 2000), ou dégrader l'état sanitaire des truies et surtout des porcelets si elles ne sont pas bien maîtrisées.

\section{a) Pendant la gestation}

Des études assez anciennes (Teague et al 1968, Omtvedt et al 1973, Mercy et Godfrey 1980) suggèrent qu'un stress thermique, au-delà de $30^{\circ} \mathrm{C}$, imposé à des truies en tout début de gestation (2 ou 8 premiers jours) réduit significativement le taux de conception. Cependant, les inséminations étant naturelles, cet effet de la chaleur peut aussi bien être lié au verrat qu'à la truie. De même, dans une étude menée en Bretagne dans 68 élevages, Leneveu (1998) rapporte une relation inverse entre la fertilité des truies, représentée par le pourcentage de truies confirmées gestantes à l'échographie, et le nombre de jours où la température ambiante excède $25^{\circ} \mathrm{C}$ pendant la semaine de saillie et les deux semaines suivantes. Une baisse de performance des verrats et la photopériode peuvent participer à la baisse de fertilité des truies en période chaude. Il est intéressant de souligner que, dans cette étude, l'effet de la chaleur est observé dans les élevages présentant des performances moyennes toute l'année, mais pas dans les élevages ayant des taux de fertilité élevés supérieurs à 85 \% (Leneveu 1998). La conduite d'élevage permettrait donc d'atténuer sensiblement les variations saisonnières de fertilité.

Des températures ambiantes supérieures à $32-34^{\circ} \mathrm{C}$ induisent une réduction de la survie embryonnaire si le stress thermique est imposé aux truies pendant la première quinzaine de gestation (Tompkins et al 1967, Edwards et al 1968, Omtvedt et al 1973, Wildt et al 1975). Au-delà de ces 15 jours, les températures élevées auraient moins d'effet sur la survie embryonnaire ou foetale, sauf durant les quinze derniers jours de gestation où elles sont de nouveau néfastes (Omtvedt et al 1973). Chez la plupart des mammifères, les températures ambiantes élevées en début de gestation peuvent être fatales au développement des embryons quand elles induisent une hyperthermie chez la mère (Vanroose et al 2000).
L'hyperthermie peut affecter directement les embryons via l'augmentation de la température au niveau utérin (Trujano et Wrathall 1986), ou indirectement en redirigeant les flux sanguins vers la périphérie du corps afin d'augmenter les pertes de chaleur et de maintenir une température corporelle adéquate, au détriment des fœtus (Dzuik 1992). Une mortalité embryonnaire modérément amplifiée par un stress thermique peut se traduire par une taille de portée réduite. Si une grande partie ou la totalité des embryons meurent, la truie avorte.

\section{b) Pendant la lactation}

Quand la température ambiante augmente au-delà de $16^{\circ} \mathrm{C}$, l'ingestion spontanée des truies décroît en moyenne de 2,4 MJ d'énergie digestible par jour et par degré (ce qui correspond environ à $170 \mathrm{~g}$ d'aliment, Black et al 1993). Cette variation n'est cependant pas linéaire et la baisse de consommation s'accentue fortement quand les températures dépassent $27^{\circ} \mathrm{C}$. Ainsi, des truies multipares maintenues à $29^{\circ} \mathrm{C}$ consomment, en moyenne sur la lactation, $30 \%$ d'aliment en moins que des truies maintenues à $27^{\circ} \mathrm{C}(3,0$ vs. $4,5 \mathrm{~kg} / \mathrm{j}$, Quiniou et Noblet 1999). Le déficit nutritionnel induit par cette baisse de consommation et la mobilisation des réserves corporelles qui en découle peuvent être à l'origine d'un anœstrus prolongé après le tarissement, notamment chez les jeunes truies (Quesnel et Prunier 1995). En effet, une plus grande variabilité du retour en oestrus après le sevrage est observée au chaud, mais seulement chez les truies primipares (Messias de Bragança et al 1998, Quiniou et al 2000).

Limiter la baisse de consommation et/ou le déficit énergétique des truies allaitantes soumises à des températures élevées est donc un enjeu important. Concentrer l'énergie des aliments de lactation en y ajoutant des matières grasses peut avoir des effets bénéfiques

Tableau 3. Influence de la température ambiante et du niveau d'alimentation chez des truies primipares (Messias de Bragança et al 1998).

\begin{tabular}{|l|c|c|c|c|}
\hline & Lot 20AL & Lot 20RA & Lot 30AL & Effet Lot \\
\hline Effectif & 8 & 8 & 8 & - \\
\hline Aliment consommé, $\mathrm{kg} / \mathrm{j}$ & $4,9 \mathrm{a}$ & $3,1 \mathrm{~b}$ & $2,8 \mathrm{~b}$ & $P<0,001$ \\
\hline Perte de poids pendant la lactation, $\mathrm{kg}$ & $-8,3 \mathrm{a}$ & $-31,5 \mathrm{c}$ & $-21,7 \mathrm{~b}$ & $P<0,001$ \\
\hline Croissance des portées, $\mathrm{kg} / \mathrm{j}$ & $2,05 \mathrm{a}$ & $1,96 \mathrm{a}$ & $1,62 \mathrm{~b}$ & $P<0,001$ \\
\hline
\end{tabular}

Les moyennes affectées d'une lettre différente sur une même ligne diffèrent à $P<0.05$.

Lot $20 \mathrm{AL}$ : truies nourries à volonté et maintenues à $20^{\circ} \mathrm{C}$; lot $30 \mathrm{AL}$ : truies nourries à volonté et maintenues à $30^{\circ} \mathrm{C}$; lot 20RA : truies maintenues à $20^{\circ} \mathrm{C}$ et rationnées de façon à consommer la même quantité d'aliment que celles du lot 30AL. sur l'intervalle sevrage-oestrus moyen (Cox et al 1983) ou non (Renaudeau et al 2001). En fait, la teneur élevée en graisse des régimes favoriserait plutôt l'adiposité des porcelets que les réserves corporelles de la truie (Tokach et Dial 1992). L’extra-chaleur produite de l'aliment puisqu'elle est plus élevée pour les protéines alimentaires que La substitution des protéines dans l'aliment par de l'amidon ou des matières grasses doit donc permettre d'augmenter la consommation des truies quand les températures dépassent la zone de confort thermique. En effet, des aliments à faible teneur en matières azotées totales (14,2 versus $17,6 \%)$ et supplémentés en acides aminés industriels atténuent les effets négatifs de la chaleur $\left(29^{\circ}\right.$ versus $\left.20^{\circ} \mathrm{C}\right)$ sur la perte de poids vif des truies pendant lactation (Renaudeau et al 2001). L'intervalle sevrage-oestrus n'est cependant pas influencé par ces régimes (Renaudeau et al 2001, Johnston et al 1999).

La sous-alimentation pendant la lactation ne mimerait cependant pas complètement l'impact des températures élevées sur les performances des truies tableau 3). Ces auteurs ont comparé des truies élevées à $30^{\circ} \mathrm{C}$ et nourries à soit nourries à volonté soit rationnées selon les quantités consommées par les truies à $30^{\circ} \mathrm{C}$. Les truies rationnées élevées à $20^{\circ} \mathrm{C}$ ont perdu beaucoup de poids mais ont assuré la croissance de leur portée. En revanche, les truies à croissance de leur portée a été plus faile. Cette moindre croissance des porpart des études. ambiante aurait donc un effet propre sur le métabolisme de la truie, avec des conséquences sur la lactation et l'activité de reproduction. La chaleur affecte pour l'amidon et les matières grasses. cont perdu moins de poids mais la 
la sécrétion d’hormones impliquées dans la thermorégulation et qui participent au métabolisme de la glande mammaire (hormones thyroïdiennes, cortisol) et induit une redistribution des flux sanguins vers la peau au détriment de la mamelle (Black et al 1993, Messias de Bragança et Prunier 1999, Renaudeau et al 2003).

\section{3 / Les facteurs qui modulent l'effet de la saison}

L'impact de la saison sur les performances de reproduction est modulé par différents facteurs liés à la conduite d'élevage (logement, alimentation) ou à la truie (génotype : Tantasuparuk et al 2000, Tummaruk et al 2000 ; réactivité émotionnelle : Wan et al 1994). Les solutions utilisables pour atténuer les effets de la chaleur sur les performances des porcs, en particulier la sélection génétique, ont récemment fait l'objet d'une synthèse (Renaudeau et al 2004).

\section{a) La présence du verrat}

La présence du verrat auprès des truies est essentielle pour limiter la baisse des performances de reproduction en été. A proximité des cochettes, elle atténue voire supprime les retards de puberté (Paterson et al 1991). A proximité des truies primipares, elle réduit la durée de l'intervalle sevrage-oestrus en été et en automne (Love et al 1993).

\section{b) Le logement des truies}

Love et al (1995) et Vesseur et al (1994a) rapportent que les truies élevées en loge individuelle pendant la gestation sont moins sujettes à la baisse de fertilité en été que les truies logées en groupe. L'absence de stress dû aux conflits sociaux et/ou l'accès à l'auge facilité pourrait expliquer l'effet protecteur du logement individuel. Cet effet confirme l'intérêt de garder les truies en logement individuel pendant le premier mois de gestation (MeunierSalaün et al 2002) et de ne les transférer en groupe qu'après 28 jours de gestation, comme cela est imposé par la directive européenne adoptée en juin 2001.

\section{c) L'alimentation en début de gesta- tion}

Un rationnement alimentaire modéré (1,5 à $2 \mathrm{~kg} /$ jour) est parfois préconisé en début de gestation, car il limiterait la mortalité embryonnaire précoce (Foxcroft 1997). Mais une interaction entre le niveau d'alimentation et la sai- son a été mise en évidence (Love et al 1995). Un niveau bas d'alimentation pendant le premier mois de gestation (1,6 à 2 kg/j) est associé à un taux de mise bas faible en été et en automne ( $<75 \%$ ) et à un taux correct (> $85 \%)$ en hiver et au printemps. En été, un niveau d'alimentation élevé (>3,6 kg/j) améliore les taux de gestation, atténuant donc l'effet saison. Virolainen et al (2004) confirment l'intérêt d'un niveau d'alimentation élevé $(>4 \mathrm{~kg} / \mathrm{j})$ en début de gestation en automne, quand les performances sont médiocres. La pratique du rationnement alimentaire en début de gestation devrait donc être limitée aux 3 ou 4 jours suivant l'insémination.

\section{d) Les traitements hormonaux}

De nombreux traitements médicamenteux ont été testés pour traiter des situations de sub-fertilité et en particulier la baisse saisonnière de fertilité. Ils agissent soit directement sur l'activité ovarienne ou utérine de la truie soit indirectement au moment de l'insémination sur les conditions favorisant le transport des spermatozoïdes et l'ovulation.

Les hormones gonadotropes (eCG + hCG) sont parfois utilisées pour stimuler l'oestrus et l'ovulation des truies. Un traitement systématique le jour du sevrage réduit le pourcentage de truies en anoestrus et augmente le pourcentage de truies qui mettent bas, quel que soit le rang de portée (Estienne et Hartsock 1998), surtout chez les primipares (Bates et al 1991, Kirkwood et al 1998) ou seulement chez les truies de rang de portée compris entre 2 et 6 (Bates et al 2000). Aux Pays-Bas, le pourcentage de truies traitées pour anœstrus après le tarissement s'élève à $17,7 \%$ en été contre $10 \%$ le reste de l’année (Vesseur et al 1994a).

L'injection intra-vulvaire d'un analogue des prostaglandines F2 $\alpha$ au moment de l'insémination, associée ou non à une injection le jour du sevrage, permettrait d'améliorer le pourcentage de truies en chaleur après le tarissement en été (Peña et al 1998a et 2001). Une augmentation de la proportion de truies qui mettent bas est également constatée, mais seulement si le taux de mise bas est particulièrement dégradé en été (< 60 \%, Peña et al 1998a).

L'addition de différentes hormones dans le sperme au moment de l'insémination a été largement testée : oestradiol, relaxine, ocytocine, prostaglandines. En stimulant les contractions utéri- nes, l'ocytocine et les prostaglandines limiteraient le refoulement des spermatozoïdes, accélèreraient leur transit vers le réservoir utéro-tubaire et augmenteraient le nombre de fœtus viables (Willenburg et al 2003). L'ajout d'ocytocine ou de prostaglandines dans la semence du verrat peut améliorer le taux de mise bas dans des élevages où les performances de reproduction sont très dégradées, avec des taux de mise bas inférieurs à 70 \% (Péña et al 1998b, Kos et Bilkei 2004). En fait, beaucoup de traitements ne s'avèrent efficaces qu'en cas de forte dégradation des résultats, et leur coût élevé justifie la recherche active des défauts de conduite à l'origine des problèmes. En effet, certaines techniques simples, telle la présence du verrat, permettent de stimuler la production naturelle des hormones recherchées, comme par exemple la décharge physiologique d'ocytocine observée chez les truies à la saillie (Langendijk et al 2003).

\section{Conclusion}

Le syndrome d'infertilité saisonnière est un problème récurrent qui n'a pas disparu malgré l'amélioration continue des performances des truies et de la maîtrise de la conduite de l'atelier de reproduction. La photopériode et la température ambiante sont à l'origine des variations saisonnières des performances de reproduction. L'existence récemment démontrée d'une sécrétion circadienne de mélatonine est en faveur d'un rôle majeur de la photopériode. Le fait que les problèmes de reproduction se prolongent bien après les périodes les plus chaudes de l'année (septembre, octobre), quand la durée d'éclairement journalière diminue le plus, et qu'ils se manifestent aussi dans des pays qui ne présentent pas de fortes chaleurs estivales (comme la Finlande) sont d'autres arguments en faveur du rôle clé de la photopériode. Cependant, des variations saisonnières des performances de reproduction sont aussi observées en zone tropicale, où la photopériode varie relativement peu entre les saisons. L'influence des températures ambiantes élevées a été clairement démontrée expérimentalement sur l'activité de reproduction des truies. Elle est en partie liée à la baisse d'appétit, chez les truies en lactation notamment, et aux adaptations métaboliques et endocriniennes mises en place par les femelles pour lutter contre l'hyperthermie.

L'impact de la saison sur la fonction de reproduction est modulé par de mul- 
tiples facteurs liés à la conduite d'élevage : alimentation des truies, conditions de logement, présence du verrat, niveau sanitaire, etc. Déterminer les points critiques de la conduite d'élevage qui permettent de réduire les problèmes d’infertilité estivale est donc un enjeu important. Enfin, la sensibilité des truies à l'effet de la saison semble varier d'un animal à l'autre, potentiellement en fonction de leur réactivité individuelle au stress.

\section{Références}

Armstrong J.D., Britt J.H., Cox N.M., 1986. Seasonal differences in function of the hypothalamic-hypophysial-ovarian axis in weaned primiparous sows. J. Reprod. Fert., 78, 11-20.

Bassett J.M., Bray C.J., Sharpe C.E., 2001. Reproductive seasonality in domestic sows kept outdoors without boars. Reproduction, 121, 613629.

Bates R.O., Day B.N., Britt J.H., Clark L.K., Brauer M.A., 1991. Reproductive performance of sows treated with a combination of pregnant mare's serum gonadotropin and human chorionic gonadotropin at weaning in the summer. J. Anim. Sci., 69, 894-898.

Bates R.O., Kelpinski J., Hines B., Ricker D., 2000. Hormonal therapy for sows weaned during fall and winter. J. Anim. Sci., 78, 2068-2071.

Belstra B. A., Flowers W.L., See M.T., 2004. Factors affecting temporal relationships between estrus and ovulation in commercial sow farms. Anim. Reprod. Sci., 84, 377-394.

Black J.L., Mullan B.P., Lorschy M.L., Giles L.R., 1993. Lactation in the sow during heat stress. Livest. Prod. Sci., 35, 153-170.

Britt J.H., Szarek V.E., Levis D.G., 1983. Characterization of summer infertility of sows in large confinement units. Theriogenology, 20, 133-140.

Chemineau P., Malpaux B., Pelletier J., Leboeuf B., Delgadillo J.A., Deletang F., Pobel T. Brice G., 1996. Emploi des implants de mélatonine et des traitements photopériodiques pour maîtriser la reproduction saisonnière chez les ovins et les caprins. INRA Prod. Anim., 9, 45-60.

Christenson R.K., 1981. Influence of confinement and season of the year on puberty and estrous activity of gilts. J. Anim. Sci., 52, 821-830.

Ciereszko A., Ottobre J.S., Glogowski J., 2000. Effects of season and breed on sperm acrosin activity and semen quality of boars. Anim. Reprod. Sci., 64, 89-96.

Clark J.R., Komkov A., Tribble F., 1986. Effects of parity, season, gonatrophin releasing hormone and altered suckling intensity on the interval to rebreeding in sows. Theriogenology, 26, 299-308.

Claus R., Schelkle G., Weiler U., 1984. [First attempts to improve sow reproductive performance in summer by means of a lighting programme]. Zuchthygiene, 19, 49-56.

Cox N.M., Britt J.H., Armstrong W.D., Alhusen H.D., 1983. Effect of feeding fat and altering weaning schedule on rebreeding in primiparous sows. J. Anim. Sci., 56, 21-29.

Cox N.M., Ramirez J.L., Matamoros I.A., Bennett W.A., Britt J.H., 1987. Influence of season on oestrous and luteinizing hormone responses to estradiol benzoate in ovariectomized sows. Theriogenology, 27, 395-405.

Cronin G.M., Hemsworth P.H., Winfield C.G., Muller B., Chamley W.A., 1983. The incidence of and factors associated with failure to mate by 245 days of age in the gilt. Anim. Reprod. Sci., 5, 199-205.

D’Allaire S., Drolet R., Brodeur D., 1996. Sow mortality associated with high ambient temperatures. Can. Vet. J., 37, 237-239.

Dawson A., Pitt R., Peters A. R., 1998. Seasonality and reproduction. In: Recent Progress in Pig Science, J. Wiseman, M.A. Varley, J.P. Chadwick (eds), 327-342, Nottingham University Press.

Dominguez J.C., Peña F.J., Anel L., Carbajo M., 1996. Swine summer infertility syndrome in North West Spain. Vet. Rec., 27, 93-94.

Dourmad J.Y., Etienne M., Prunier A., Noblet J., 1994. The effect of energy and protein intake of sows on their longevity: a review. Livest. Prod. Sci., 40, 87-97.

Dziuk P.J., 1992. Embryonic development and fetal growth. Anim. Reprod. Sci., 28, 299-308.

Edwards R.L., Omtvedt I.T., Turman E.J., Stephens D.F., Mahoney G.W.A., 1968. Reproductive performance of gilts following heat stress prior to breeding and in early gestation. J. Anim. Sci., 27, 1634-1638.

Elbers A.R.W., van Rossem H., Scukken Y.H., Martin S.W., van Exsel A.C.A., Friendship R.M., Tielen M.J.M., 1994. Return to oestrus after first insemination in sow herds (incidence, seasonality, and association with reproductivity and some blood parameters). Vet. Quart., 16, 100-109.

Estienne M.J., Hartsock T.G., 1998. Effect of exogenous gonadotropins on the weaning-toestrus interval in sows. Theriogenology, 49, 823828 .

Flowers B., Cantley TC, Martin M.J., Day B.N., 1989. Effect of elevated ambient temperatures on puberty in gilts. J. Anim. Sci., 67, 779785.

Foxcroft G.R., 1997. Mechanisms mediating nutritional effects on embryonic survival in pigs. J. Reprod. Fert., Suppl. 52, 47-61.

Gaustad-Aas A.H., Hofmo P.O., Karlberg K., 2004. Reproductive performance of gilts and sows in temperate versus subarctic and arctic zones of Norway. Anim. Reprod. Sci., 80, 291301.

Guillouet P., Tribout T., Bussière J.F., Bertaud G., Bidanel J.P., Terqui M., 1999. Analyse des facteurs de variation de la production spermatique de verrats d'insémination artificielle. Journ. Rech. Porcine Fr., 31, 45-52.

Hennessy D.P., Williamson P.E., 1984. Stress and summer infertility in pigs. Austr. Vet. J., 61, 212-215.

Hochereau de Reviers M.T., Lagant H., Després P., Venturi E., Brunet L., Perreau C., Wianny F., Legault C., 1997. Facteurs de variation de l'âge à la puberté, du taux d'ovulation et de la survie embryonnaire précoce chez la cochette : type génétique, poids vif, saison, et consanguinité. Journ. Rech. Porcine Fr, 29,109114.

Hoste S., Oakenfull A., 1999. A genetic approach to seasonal infertility in pigs. In: Pig Reproduction: Problems, Practices and Principles, R.H.C. Penny, D.H. Machin (eds), 86-93, Cambac associates publication.

Huang S.Y., Kuo Y.H., Lee Y.P., Tsou H.L., Lin E.C., Ju C.C., Lee W.C., 2000. Association of heat shock protein 70 with semen quality in boars. Anim. Reprod. Sci., 63, 231-240.

Johnston L.J., Ellis M., Libal G.W., Mayrose V.B., Weldon W.C., NCR-89 Committee on swine management, 1999. Effect of room temperature and dietary amino acid concentration on performance of lactating sows. J. Anim. Sci., 77, 1638-1644.

Karlberg K., 1980. Factors affecting postweaning oestrus in the sow. Nord. Vet. Med., 32, 185-193.

Kirkwood R.N., Aherne F.X., Foxcroft G.R., 1998. Effect of gonadotropin at weaning on reproductive performance of primiparous sows. Swine Health Prod., 6, 51-55.

Koketsu Y., Dial G.D., King V.L., 1997. Returns to service after mating and removal of sows for reproductive reasons from commercial swine farms. Theriogenology, 47, 13471363

Kos M, Bilkei G., 2004. Prostaglandin F2 supplemented semen improves reproductive performance in artificially inseminated sows. Anim. Reprod. Sci., 80, 113-120.

Langendijk P., Bouwman E.G., Schams D. Soede N.M., Kemp B., 2003. Effects of different sexual stimuli on oxytocin release, uterine activity and receptive behaviour in estrous sows. Theriogenology, 59, 849-861.

Le Cozler Y., Dagorn J., Dourmad J.Y., Johansen S., Aumaître, A., 1997. Effect of weaning-to-conception interval and lactation length on subsequent litter size in sows. Livest. Prod. Sci., 51, 1-11.

Leman A.D., 1992. Optimizing farrowing rate and litter size and minimizing nonproductive sow days. Vet. Clinics of North America: Food Animal Practice, 8, 609-621.

Leneveu P., 1998. Suivi échographique de 68 élevages de truies sur 14 mois. Résultats, analyse et étude sur l'infertilité d'été en fonction de la température ambiante. Journ. Rech. Porcine Fr., 30, 423-427.

Levis D.G., 2002. What's new with seasonal infertility? Ohio Pork Industry Center, the Ohio state university extension. www.porkinfo. osu.edu.

Love R.J., Evans G., Klupiec C., 1993 Seasonal effects on fertility in gilts and sows. J. Reprod. Fertil., Suppl. 48, 48, 191-206.

Love R.J., Klupiec C., Thornton E.J., Evans G., 1995. An interaction between feeding rate 
and season affects fertility of sows. Anim. Reprod. Sci., 39, 275-284.

McGlone J.J., Stansbury W.F., Tribble L.F., 1988. Management of lactating sows during heat stress: effects of water drip, snout coolers, floor type and a hygh energy-density diet. J. Anim. Sci., 66, 885-891.

Malpaux B., Migaud M., Tricoire H., Chemineau P., 2001. Biology of mammalian photoperiodism and the critical role of the pineal gland and melatonin. J. Biol. Rhythms, 16, 336-347.

Martinat-Botté F., Dagorn J., Terqui M., Dando P., 1984. Effect of confinement, climatic conditions and litter parity on the seasonal variations of the fertility rate and prolificacy. Ann. Rech. Vet., 15, 165-172.

Martinat-Botté F., Quesnel H., Prunier A., Tournut J., Terqui M., 1996. Reproduction de la truie : bases physiologiques et maîtrise. 1ère partie. Rev. Méd. Vét., 147, 33-46.

Messias de Bracança M., Mounier A.M., Prunier A., 1998. Does feed restriction mimic the effect of increased ambient temperature in lactating sows? J. Anim. Sci., 76, 2017-2024.

Messias de Bragança M., Prunier, A., 1999. Effects of low feed intake and hot environment on plasma profiles of glucose, nonesterified fatty acids, insulin, glucagon, and IGF-I in lactating sows. Domest. Anim. Sci., 19, 89-101.

Mercy A.R., Godfrey N.W., 1980. The effects of high ambient temperatures on the reproductive performance of gilts. Proc. Austr. Soc. Anim. Prod., 13, 393-396.

Meunier-Salaün M.C., Courboulay V., Père M.C., Pol F., Quesnel H., 2002. Elevage des truies gestantes en groupe : acquis et perspectives de recherches. Journ. Rech. Porcine Fr., 34, 239-247.

Muirhead M.R., Alexander T.J.L., 2000. The management of infertility. In: A pocket guide to recognising and treating pig infertility. 5M Enterprises Ltd Editors. 203p.

Omtvedt I.T., Nelson R.E., Edwards R.L., Stephens D.F., Turman E.J., 1973. Influence of heat stress during early, mid and late pregnancy of gilts. J. Anim. Sci., 32, 312-317.

Park C.S., Yi Y.J., 2002. Comparison of semen characteristics, sperm freezability and testosterone concentration between Duroc and Yorkshire boars during seasons. Anim. Reprod. Sci., 73, 53-61.

Paterson A.M., Pearce G.P., 1990. Attainment of puberty in domestic gilts reared under longday or short-day artificial light regimens. Anim. Reprod. Sci., 23, 135-144.

Paterson A.M., Pearce G.P., Antuono M.F.D., 1991. Seasonal variation in attainment of puberty in isolated and boar-exposed domestic gilts. Anim. Reprod. Sci., 24, 323-333.

Peltoniemi O.A.T., Love R.J., Heinonen M., Tuovinen V., Saloniemi H., 1999. Seasonal and management effects on fertility of the sow: a descriptive study. Anim. Reprod. Sci., 55, 47-61.

Peltoniemi O.A.T., Tast A., Love R.J., 2000. Factors affecting reproduction in the pig: seasonal effects and restricted feeding of the pregnant gilt and sow. Anim. Reprod. Sci., 6061, 173-184.

Peña F.J., Dominguez J.C., Alegre B., Pelaez J., 1998a. Effect of vulvomucosal injection of
PGF2 $\alpha$ at insemination on subsequent fertility and litter size in pigs under field conditions. Anim. Reprod. Sci., 52, 63-69.

Peña F.J., Dominguez J.C., Carbajo M., Anel L., Alegre B., 1998b. Treatment of swine summer infertility syndrome by means of oxytocin under fields conditions. Theriogenology, 49, 829-836.

Peña F.J., Gil M.C., Peña F., 2001. Effect of vulvomucosal injection of cloprostenol at weaning and at insemination on reproductive performance of sows during the low fertility summer season under field conditions. Anim. Reprod. Sci., 68, 77-83.

Prunier A., Dourmad J.Y, Etienne M., 1994. Effect of light regimen under various temperatures on sow and litter performance. J. Anim. Sci., 72, 1461-1466.

Quesnel H., Prunier A., 1995. Endocrine bases of lactational anoestrus in the sow. Reprod. Nutr. Develop., 35, 395-414.

Quesnel H., Martinat-Botté F., Prunier A., Tournut J., Terqui M., 1996. Reproduction de la truie : bases physiologiques et maîtrise. $2^{\mathrm{e}}$ partie. Rev. Méd. Vét., 147, 111-122.

Quiniou N., Noblet J., 1999. Influence of high ambient temperatures on performance of multiparous lactating sows. J. Anim. Sci., 77, 21242134.

Quiniou N., Gaudré D., Rapp S., Guillou D., 2000. Influence de la température ambiante et de la concentration en nutriments de l'aliment sur les performances de lactation de truies primipares. Journ. Rech. Porcine Fr., 32, 275-282.

Renaudeau D., Noblet J., Quiniou N., Dubois S., 2001. Influence de l'exposition au chaud et de la réduction du taux en protéines dans l'aliment sur les performances des truies multipares en lactation. Journ. Rech. Porcine Fr., 33, 181187.

Renaudeau D., Noblet J., Dourmad JY., 2003. Effect of ambient temperature on mammary gland metabolism in lactating sows. J. Anim. Sci., 81, 217-231.

Renaudeau D., Mandonnet N., TixierBoichard M., Noblet J., Bidanel J-P, 2004. Atténuer les effets de la chaleur sur les performances des porcs : la voie génétique. INRA Prod. Anim., 17, 93-108.

Rozeboom, K.J., Todd See, M., Flowers, W., 2000. Management practices to reduce the impact of seasonal infertility on sow herd productivity. Anim. Sci. Facts, ANS-813S, 10 p.

Steverink D.W,. Soede N.M., Groenland G. J, van Schie F.W., Noordhuizen J.P, Kemp B., 1999. Duration of estrus in relation to reproduction results in pigs on commercial farms. J. Anim Sci., 77, 801-809.

Tantasuparuk W., Lundeheim N., Dalin A-M., Kunavongkrit A., Einarsson S., 2000. Reproductive performance of purebred Landrace and Yorkshire sows in Thailand with special reference to seasonal influence and parity number. Theriogenology, 54, 481-496.

Tast A., Hälli O., Ahlström S., Adersson H., Love R.J., Peltoniemi O.A.T., 2001. Seasonal alterations in circadian melatonin rhythms of the European wild boar and domestic gilt. J. Pineal Res., 30, 43-49.

Tast A., Peltoniemi O. A. T., Virolainen J.V., Love R.J., 2002. Early disruption of pregnancy as a manifestation of seasonal infertility in pigs. Anim. Reprod. Sci., 74, 75-96.

Teague H.S., Roller W.L., Grifo A.P. Jr., 1968. Influence of high temperature and humidity on the reproductive performance of swine. J. Anim. Sci., 27, 408-411.

Tokach M.D., Dial G.D., 1992. Managing the lactating sows for optimal weaning and rebreeding performance. Vet. Clinics of North America: Food Animal Practice, 8, 559-574.

Tompkins E.C., Heidenreich C.J., Stob M., 1967. Effect of post-breeding thermal stress on embryonic mortality in swine. J. Anim. Sci., 26, 377-380.

Tubbs R.C., 1990. Factors that influence the weaning to oestrus interval in sows. The compendium, Food Anim., 12, 105-115.

Trujano M., Wrathall A.E., 1986. Effects of hyperthermia on cultured explants of porcine endometrium. British Vet. J., 142, 141-144.

Tummaruk P., Lundeheim N., Einarsson S., Dalin A.M., 2000. Reproductive performance of purebred Swedish Landrace and Swedish Yorkshire sows: I. Seasonal variation and parity influence. Acta Agric. Scand., Sect. A., Anim. Sci., 50, 205-216.

Tummaruk P., Lundeheim N., Einarsson S., Dalin A.M., 2001. Reproductive performance of purebred Hampshire sows in Sweden. Livest. Prod. Sci., 68, 67-77.

Vanroose G., de Kruif A., Van Soom A, 2000. Embryonic mortality and embryo-pathogen interactions. Anim. Reprod. Sci., 60-61, 131143.

Vesseur P.C., Kemp B., Den Hartog L.A., 1994a. Factors affecting the weaning to estrus interval in the sow. J. Anim. Physiol. a. Anim. Nutr., 72, 225-233.

Vesseur P.C., Kemp B., Den Hartog L.A., 1994b. The effect of the weaning to oestrus interval on litter size, live born piglets and farrowing rate in sows. J. Anim. Physiol. a. Anim. Nutr. 71, 30-38.

Virolainen J.V., Tats A., Sorsa A., Love R.J., Peltoniemi O.A.T., 2004. Changes in feeding level during early pregnancy affect fertility in gilts. Anim. Reprod. Sci., 80, 341-352.

Wan S.S., Hennessy D.P., Cranwell P.D., 1994. Seasonal infertility, stress and adrenocortical responsiveness in pigs. Anim. Reprod. Sci., 34, 265-279.

Wildt D.E., Riegle G.D., Dukelow W.R., 1975 Physiological temperature response and embryonic mortality in stressed swine. Am. J. Physiol., 229, 1471-1479.

Willenburg K.L., Miller G., Rodriguez-Zas S.L., Knox R.V., 2003. Influence of hormone supplementation to extended semen on artificial insemination, uterine contractions, establishment of sperm reservoir, and fertility in swine. $\mathrm{J}$. Anim. Sci., 81, 821-829.

Wrathall A.E., Wells D.E., Jones P.C., Foulkes J.A., 1986. Seasonal variations in serum progesterone levels in pregnant sows. Vet. Rec., 118, 685-687.

Xue J.L., Dial G.D., Marsh W.E., Davies P.R., 1994. Multiple manifestations of season on reproductive performance of commercial swine. J. Am. Vet. Med. Assoc., 204, 1486-1489. 


\section{Résumé}

Bien que la truie ne connaisse pas d'anœstrus saisonnier, une diminution des performances de reproduction est observée en été et au début de l'automne dans de nombreux pays. La proportion de truies qui tardent à revenir en oestrus après le tarissement augmente tandis que la proportion de truies inséminées qui mettent bas baisse de 5 à $15 \%$. Ces échecs de gestation sont liés, pour une grande part, à des avortements. Cette baisse des performances peut s'accompagner d'un taux de réforme accru, notamment chez les jeunes femelles.

L'influence des saisons est attribuée essentiellement à la photopériode et aux températures ambiantes. La durée quotidienne d'éclairement est perçue par la truie via la sécrétion nocturne de mélatonine par la glande pinéale, comme chez les mammifères à reproduction saisonnière. Cependant, le lien entre mélatonine et sécrétion de LH n'est pas établi chez la truie. La chaleur, si elle induit une hyperthermie chez la mère, affecte la survie des embryons en début de gestation. Pendant la lactation, les truies luttent contre l'hyperthermie en réduisant leur consommation d'aliment et développent des adaptations métaboliques et endocriniennes. Le déficit nutritionnel est connu pour favoriser le risque d'anoestrus après le tarissement. Les adaptations physiologiques liées à la chaleur joueraient aussi un rôle.

La conduite d'élevage module les variations saisonnières des performances de reproduction : alimentation et logement des truies, présence du verrat, etc. Une meilleure caractérisation des points critiques dans la gestion de l'atelier de reproduction devrait permettre d'atténuer ces fluctuations saisonnières. Enfin, l’origine de la sensibilité individuelle à la saison mériterait d'être étudiée.

\section{Abstract}

\section{Seasonal variation of reproductive performance of the sow}

The sow is not a seasonal breeder. However, reproductive performance decreases during the summer and early fall in many countries. The proportion of sows with a delayed oestrus after weaning increases and the proportion of inseminated sows that do not farrow is reduced by 5 to $15 \%$. Pregnancy failure is largely due to abortion. Reproductive dysfunction can be associated to a higher culling rate, especially for young sows.

The influence of season is mainly attributed to photoperiod and ambient temperature. The daily light duration is perceived by the sow through melatonin secretion by the pineal gland, as in all seasonal breeders. However, the link between melatonin and LH secretion has not yet been demonstrated in the sow. Heat stress can alter embryo survival in early gestation through sow hyperthermia. During lactation, sows adapt to high temperatures by reducing feed intake and adjusting metabolic and endocrine status. Nutritional deficit is known to increase the risk of anoestrus after weaning. Physiological adaptations to heat stress are likely to also play a role.

Herd management modulates seasonal variations of reproductive performance: sow nutrition and housing, presence of the boar... A better understanding of the critical points in the management of reproductive sows may help attenuate seasonal fluctuations. In addition, the origin of the individual differences in responsiveness to the season effect needs further investigation.

QUESNEL H., BOULOT S., LE COZLER Y. 2005. Les variations saisonnières des performances de reproduction chez la truie. INRA Prod. Anim., 18, 101-110. 